\title{
Developmental changes in free recall and serial learning of categorically structured lists
}

\author{
CARLA J. POSNANSKY \\ University of Rochester, Rochester, New York 14627 \\ and \\ JAMES W. PELLEGRINO \\ University of Pittsburgh, Pittsburgh, Pennsylvania 15203
}

\begin{abstract}
Free recall performance with both constant and varied input orders was compared to serial recall performance across three grade levels. Categorized lists composed of frequent instances from categories familiar to young children were presented under both blocked and nonblocked conditions. Results revealed high levels of recall performance and semantic clustering with 'no evidence of production deficiency. Both the amount recalled and the number of instances per category increased with increasing grade level, while the degree of clustering did not. Serıal and tree recall performance did not differ due to the high use of semantic clustering in free recall, which made the previously facilitative provision of an ordering strategy during serial learning no longer useful.
\end{abstract}

Developmental assessments of free recall (FR) learning have generally reported relatively low overall performance levels and corresponding low organizational scores for clustering and/or subjective organization (e.g., Laurence, 1966, 1967). Jablonski (1974) has suggested that organization in the FR learning of children should be diminished to the degree that a large verbal repertoire has not yet developed. Additionally, Nelson (1969) has postulated that poor performance on categorized lists may result from children's conceptual categories containing instances which differ from those in adult categories.

An alternative, but compatible, explanation of the low recall and organizational levels has been offered by Flavell (1970) with the formulation of a production deficiency hypothesis. The latter proposes that the poor performance is due to a failure to produce semantic mediators during storage and/or retrieval. Such a failure may be due to the reduced development of the verbal repertoire and/or the differences in adult and children's conceptual hierarchies. However, a production deficiency need not depend upon either factor.

The production deficiency explanation of FR also fits well with developmental differences in serial and FR performance. Posnansky (1974a) has noted that children exhibit high performance levels during serial learning (SL) as compared to typical FR. For example, Posnansky showed that children as young as first graders could master a 9-item serial list in an average of six trials, while in Nelson's (1969) free-recall study, second-grade

Requests for reprints should be sent to the first author, care of the College of Education, University of Rochester, Rochester. New York 14627. The authors are grateful to Dorothy Leuze for her help in data collection. The authors are grateful to the principal and faculty of Brookside Elementary School. children were recalling an average $49 \%$ of the 15 -item list after 15 trials. The nature of the serial task in its provision of a study and retrieval scheme may well serve to overcome the existence of a production deficiency by the substitution of a serial ordering strategy for both storage and retrieval. However, developmental differences in SL and FR learning may not be due to the provision of a storage and retrieval ruie, per se, but rather an inherent sensitivity to serial structure which is not tapped by the typical constantly varying orders in FR. Serial structure (contiguity of occurrence) may be a more important organizational scheme than semantic structure (see Jablonski, 1974) and this may explain low performance levels in FR and the superiority of SL in young children.

The present study attempted to contrast these varying explanations of developmental SL and FR performance by directly comparing the two tasks on both blocked and nonblocked categorized lists under FR conditions where list order either remained constant or changed across successive trials. If there is a production deficiency in the use of semantic mediators and this is not overcome by the use of children's normative category instances, then SL should be superior to FR with varying list orders. Similarly, if children are also not sensitive to serial constancy then SL should also be superior to constant order FR. However, if serial structure is more important than semantic structure for young children then constant order FR may be equal to SL and both would be superior to varied order FR. Finally, if the use of children's normative category instances serves to overcome the aforementioned difficulties in previous studies of semantic organization, then both constant and varied order FR should be equal in performance and both should be superior to SL. To 
the extent that semantic blocking also serves to overcome any production deficiency, (e.g., Cole, Frankel, \& Sharp, 1971) the difference between FR and SL may be eliminated under blocking conditions while SL would be superior under nonblocked presentation.

\section{METHOD}

\section{Subjects}

The subjects in the present study were 48 first graders, 48 third graders, and 36 fourth graders at Brookside elementary school in Rochester, New York. Mean ages of the subjects were 6 years 7 months, 8 years 7 months, and 9 years 8 months, respectively.

\section{Design and Procedure}

The present design was a 3 by 3 by 2 factorial manipulating grade level (1, 3, and 4), learning task (serial, constant order FR and varying order FR), and blocking (blocked and nonblocked presentation). The subjects in each grade were tested individually and randomly assigned to experimental cells so that there were eight subjects in each cell in Grades 1 and 3 and six subjects in each cell in Grade 4. Two-thirds of the subjects received FR instructions which informed them that they would see several pictures which they should try their best to remember, while one-third of the subjects received serial recall instructions which included the further directive to recall the items in order. The subjects were informed that they would have several opportunities to see and recall the list. The subjects had eight trials with one-half of the FR subjects seeing the list in the same order on each trial (constant order FR) while the other FR subjects saw the list in a different order on each trial (varied order FR). Trial number and list order were varied in a Latin square to balance list orders across conditions. Within each of the three learning tasks, one-half of the subjects received a nonblocked list while the other half received blocked lists.

The items on study trials were presented at a 3-sec rate with 1 -min of oral recall. Intertrial intervals consisted of only the few seconds needed by the experimenter to prepare for the next trial.

\section{Materials \\ Four frequently occurring instances of four different categories (animals, items of clothing, parts of the body, fruits) were selected from Posnansky's (1974b) children's category norms with the restriction that all items could be easily depicted in simple black and white line drawings. Eight distinct list orders were randomly prepared with the restriction that no two items from the same semantic category ever appeared together in adjacent serial positions. These list orders were used for the nonblocked experimental conditions. Eight other list orders were prepared so that all items from a category appeared contiguously but so that order within and between categories was never repeated in more than one list order. These list orders were used in the blocked experimental conditions. Lists were presented on $3 \times 5$ cards placed within a photograph album.}

\section{RESULTS}

\section{Recall}

Results indicated that the mean number of items recalled per trial was equal for the serial recall, and constant order and varied order FR tasks (means of $11.15,11.31$, and 11.10 , respectively), $\mathrm{F}<1$. However, in SL when the number of items recalled in either the correct serial position or following the correct preceding item was used as the dependent measure (rather than absolute number recalled) an average of one item less per trial was recalled. As can be seen in Figure 1, recall increased across grade levels, $\mathrm{F}(2,110)=59.93$, $\mathrm{p}<.001$, with means of $9.16,11.71$, and 12.69 for grades 1, 3, and 4, respectively. Additionally, more items were recalled per trial under the blocked (11.80) than nonblocked (10.57) conditions in all three tasks, $F(1,110)=20.50, p<.001$. No other effects or interactions approached significance, $\mathrm{ps}>.10$, except for the effect of trials.

Recall on each trial was broken down into the number of categories and items per category represented. The analysis of number of categories showed no effects, Fs $<1.5$. The analysis of items per category showed a grade level effect, $F(2,110)=31.95, \mathrm{p}<.001$ with means of $2.59,3.06$, and 3.27 for grades 1,3 , and 4 respectively. As in the analysis of overall recall, there was a blocking effect, $\mathrm{F}(1,110=8.74, \mathrm{p}<.01$, with blocked (2.95) superior to unblocked (2.62) presentation. No other effects or interactions approached significance, ps $<.10$, except for trials.

\section{Organization}

Clustering. Analysis of variance using the Roenker, Thompson, and Brown (1971) ARC index of semantic clustering as the dependent measure provided an overall mean ARC score of .634 . Higher scores were obtained under blocked (.721) than nonblocked (.546) conditions, $F(1,76)=9.05, p<.01$. There were no differences in the amount of clustering across the three grades, $F=1.02$, or across constant and varied presentation conditions, $F=1.07$. No other effects or interactions approached significance except for trials. See Figure 1.

Seriation. $\mathrm{ARC}_{\alpha / \chi}^{\gamma}$ scores (Pellegrino, 1971) for input-output consistency were also examined. Two unit sizes (two and three) were scored using the unordered criterion. An analysis of variance here revealed only larger scores under blocked $(\overline{\mathrm{X}} \mathrm{s}=.333$ and .235$)$ then nonblocked conditions $(\overline{\mathrm{X}} \mathrm{s}=.016$ and .001), $\operatorname{Fs}(1,84)>66.99, \mathrm{p}<.001$.

These results indicated that the degree of seriation was reflecting semantic clustering rather than seriation as an independent output strategy.

Priority. Priority in the recall of newly learned items was assessed by using the relative index of priority measure (RIP) (Flores \& Brown, 1974). No effects or interactions were significant $(\mathrm{ps}<.10)$ and the mean score for new items was .029 which was not significantly above zero $(\mathrm{F}<1)$.

Primacy and recency. The items comprising the primacy and recency portions of each trial input order were examined for output recall position using the RIP measure. The items in the last quarter of the list (.18) were recalled earlier than items in the first quarter $(-.04), F(1,76)=23.85, p<.001$. No other effects or 

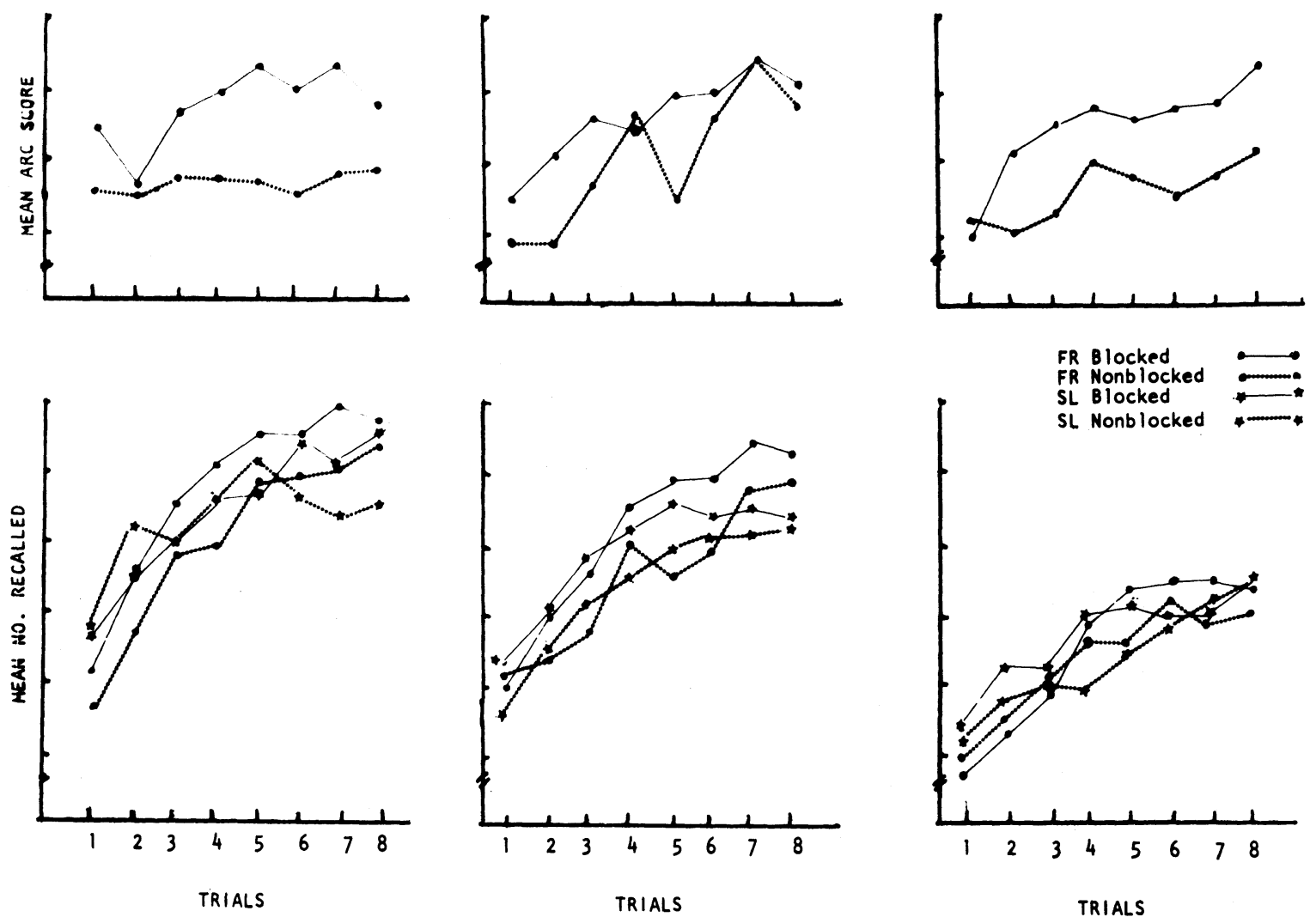

GRADE 4

GRADE 3

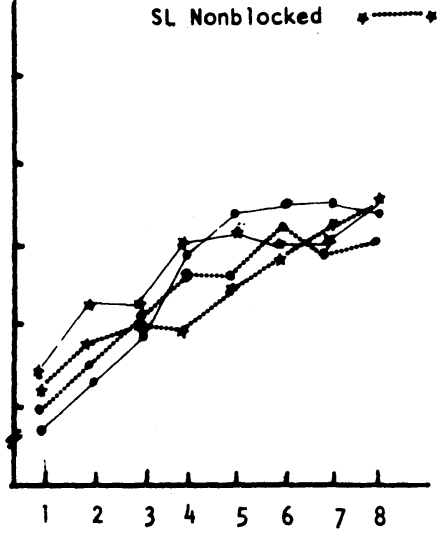

TRIALS

GRADE 2

interactions approached significance (ps $>.10)$.

\section{DISCUSSION}

The present data are clear with respect to the various hypotheses advanced in the introduction. FR performance levels at all age levels were relatively high for both recall and semantic clustering with little evidence in support of a production deficiency in younger children. Additionally, this conclusion applied to both blocked and nonblocked item presentation. The unusually high level of clustering predominated over other kinds of organization including seriation. Such a pattern of results in categorized lists is consistent with adult performance (e.g., Pellegrino \& Battig, 1974), including relatively low levels of new item priority and primacy-recency output strategies. Such strategies are reduced when a high level of semantic organization is present, and, thus, the present organization results further support the lack of evidence for a production deficiency in the present data, even among the youngest children. The present data also argue that semantic structure is used in preference to serial constancy and that even in young children the semantic basis of organization predominates.

The overall recall levels in the present study were also quite high when viewed in light of Jablonski's (1974) recent summary which stated that many developmental studies have failed to report more than $50 \%$ recall regardless of experimental manipulations. Both the high recall performance and the high degree of semantic clustering may be attributable to the use of highly familiar semantic categories which were capable of eliciting many instances from children (see Posnansky, 1974b) and which, therefore, involve familiar superordinate labels. The present study also utilized instances from those categories with high normative frequencies for children and Jablonski (1974) has emphasized the potential developmental importance of both these factors. Finally, the use of pictures in the present study may also have contributed to the high degree of category clustering and recall (e.g., Cole et al., 1971). Children's use of imagery may make the common semantic referent more apparent since a given image may suggest both the instance and category label. The use of pictures may also lead to the circumvention of acoustic or phonetic processing thereby providing more rapid access to semantic levels of processing.

To the extent that previous investigations of clustering in young children have shown poor performance and were due to production deficiencies, the present lack of clustering differences across ages is most appropriately viewed as the result of improvement at the youngest ages, i.e., the elimination of production deficiencies through the previously described mechanisms. However, there was an effect of age level upon amount recalled. Such recall differences without corresponding clustering differences have been observed before (e.g., Horowitz, 1969) and could be interpreted as supporting the independence of organization and recall in children. However, such an interpretation is probably mistaken for a number of reasons. Pellegrino and Battig (1974) have argued that relationships between organization and recall cannot be assessed by any single organization index. Colle (1972) has further argued that questions about the interdependence between organization and recall cannot be assessed by current clustering indices and that such a problem depends upon a well defined theory of clustering or organization. Finally, the presence or absence of correlations between organization and recall may depend upon the particular 
metric used. The present study has used a ratio measure which expresses organization as a function of the observed deviation from chance over the maximum possible deviation given the total amount recalled. Such a measure is relatively independent of total recall and recall and organization need not be correlated across ages. The present item per category differences plus equivalent clustering scores indicate that the items recalled were equivalently organized in a semantic retrieval plan but that the age differences reflected capacity limits in the development or use of that retrieval plan. With younger children the effectiveness of the superordinate category label may be limited to fewer total instances while these limits are reduced in older children. Such a pattern would be consistent with the lack of an organizational difference but overall total recall differences. This also does not argue that recall and organization are independent in young children.

Since the present FR results indicate no significant evidence for production deficiencies. the lack of a difference between $F R$ and SL is readily interpretable. Differences between the two tasks would only be expected if semantic organization was not spontaneously used by the younger children. Thus the present equivalence of $F R$ and $S L$ can be taken as a further indication that previous demonstrations of superior SL in children compared to FR were in fact due to production deficiences in FR which were eliminated by the nature of the SL task.

In summary, the present results do not argue against a production deficiency explanation of developmental FR and SL learning but instead point out a case in which production deficiencies did not occur. presumably due to the presence of a very salient semantic structure. This suggests that previous developmental assessments of clustering in FR (e.g.. Jablonski. 1974) may have underestimated the ability of young children to produce and use semantic retrieval schemes because the schemes available were defined by adults rather than made contingent on children's own responses.

\section{REFERENCES}

Cole, M., Frankel, F., \& Sharp, D. Development of free recal learning in children. Developmental Psychology, 1971, 4, 109-123.

Colle, H. A. Reification of clustering. Journal of Verbal Learning and Verbal Behavior, 1972, 11,624-633.

Flavell, J. H. Developmental Studies of Mediated Memory. In H. W. Reese \& L. P. Lipsitt (Eds.), Advances in child development and behavior. Vol. 5, New York: Academic Press, 1970.

Flores, L. M. \& Brown, S. C. Comparision of measures of output order in free recall. Behavioral Research Methods \& Instrumentation, $1974,6,385-388$.

Horowitz, A. B. Effects of stimulus presentation on children's recall and clustering. Psychonomic Science, 1969, 14, 297-298.

Jablonski, E. M. Free recall in children. Psychological Bulletin, $1974,81,522-539$

Laurence, M. W. Age differences in performance and subjective organization in the free recall learning of pictorial material. Canadian Journal of Psy chology, 1966, 20, 388-399.

Laurence, M. W. A developmental look at the usefulness of list catagorization as an aid of free recall. Canadian Journal of Psychology, 1967, 21, 153-156.

Nelson, K. J. The organization of free recall by young children. Journal of Experimental Child Psychology, 1969, 8, 284-295.

Pellegrino, J. W. A general measure of organization in free recall for variable unit size and internal sequential consistency. Behavior Research Methods \& Instrumentation, 1971, 3, 241-246.

Pellegrino, J. W., \& Battig, W. F. Relationships among higher order organizational measures and free recall. Journal of Experimental Psy chology, 1974, 102, 463-472.

Posnansky, C.J. An investigation of serial learning and retention processes in children. Journal of Experimental Child Psy chology, 1974,18,127-148.(a)

Posnansky, C. J. Category norms of children, Behavorial Research Methods \& Instrumentation, 1974, 6, 373. (b)

Roenker, D. L., Thompson, C. P., \& Brown, S. C. A comparison of measures for the estimation of clustering in free recall. Psy chological Bulletin, 1971, 76, 45-48.

(Received for publication November $11,1974$. ) 